\title{
Feature Matching Method for Aircraft Positioning on Airdrome
}

\author{
Jian Wang ${ }^{1(\star 凶)}$ and Yubo $\mathrm{Ni}^{2}$ \\ ${ }^{1}$ Civil Aviation ATM Institute, Civil Aviation University of China, \\ Tianjin 300300, China \\ caucwang@263. net \\ ${ }^{2}$ Sino-European Institute of Aviation Engineering, Civil Aviation University of China, \\ Tianjin 300300, China
}

\begin{abstract}
The binocular stereoscopic vision technology could be used to locate targets on an aerodrome. To ensure positioning targets, the pixels on images from two vision sensors should be matched accordingly. Combining advantages of both SIFT (Scale-invariant feature transform) and an epipolar line constraint equation, a method of pixel matching is proposed. Roughly-matched points are first obtained using SIFT, in which some mismatched ones exist. Matched points are different from mismatched ones when an epipolar line constraint equation is introduced. Although matched points do not always meet the epipolar line constraint equation because of system measuring errors, the difference between the values of a matrix and critical threshold could be used to distinguish the mismatched pixels from roughly matched ones. This method can not only ensure the accuracy for the target matching, but also automatically find out feature points of the aircraft used for positioning.
\end{abstract}

Keywords: Binocular vision $\cdot$ SIFT $\cdot$ Epipolar line constraint $\cdot$ Mismatched points $\cdot$ Fundamental matrix $\cdot$ Threshold

\section{Introduction}

The airport complex operating environment needs means of surface surveillance to acquire transport traffic situations [1]. Nowadays, for hub airport surface surveillance, the S-mode radar and multi-point positioning sensors are used for the surveillance of cooperative targets. ICAO's (International Civil Aviation Organization) Advanced-Surface Movement Guidance and Control System (A-SMGCS) requires surveillance tools for non-cooperative targets [2]. Binocular stereo vision technology with good positioning accuracy has established the mathematical relationship between targets and their images. Using this technique, matching of targets of similar characteristics is important.

J. Wang-Professor, supervisor of postgraduate, main research field for high speed optical fiber communication system, photoelectric detection technology, air traffic control radar display technology. 
In the field of image processing, methods of target matching based on image features have their own advantages, with good adaptability on the gray scale, the strain and the block [3]. Among these methods, the Scale Invariant Feature transform (SIFT) algorithm proposed by David Lowe has good robustness to most of the noise, brightness changes disturbances, etc. [3]. Although it can be used as a target matching method for the binocular stereo vision applications, it needs to be improved when used as a method for positing targets, since SIFT would produce easily error matching points. A proposal is put forwards by introducing an epipolar line constraint to remove mismatched points.

\section{Matching Algorithm}

\subsection{SIFT}

There are three main steps for SIFT: (1) detecting scale-space's extremas, localizing accurately key-points; (2) assigning orientation and establishing local image descriptors; (3) matching key-points.

The scale space of an image is defined as a function, which is generated from the convolution of a variable-scale Gaussian $\mathrm{G}(\mathrm{x}, \mathrm{y}, \sigma)$ with an input image of $\mathrm{I}(\mathrm{x}, \mathrm{y})$ [4]:

$$
L(x, y, \sigma)=G(x, y, \sigma) * I(x, y)
$$

where, $(x, y)$ is the position on an image, $\sigma$ represents the degree of a smoothing scale parameter, showing the feature of an image. When the scale parameter is great, it can show the detail of the image. When the scale parameter is small, it can show the general information of the image [5]. To achieve rotation invariance and a high level of efficiency in scale space, the key location is selected at the maxima or the minima value of the difference of a Gaussian function [6], meaning that $\frac{\partial}{\partial \sigma}\left(\sigma^{2} \nabla^{2} L\right)=0$. The efficient way of detecting keypoint locations in scale space is applying scale-space's extrema to the Difference-of-Gaussian (DoG) function convolved with the image. As DoG function has a strong response along edges, the edge response must be eliminated [7].

By assigning a consistent orientation to each keypoint based on local image features, the keypoint descriptor can be represented relative to this orientation and therefore achieve invariance to image rotation [8]. or image sampling on a certain scale, the gradient magnitude $m(x, y)$ and orientation $\theta(x, y)$ are computed using pixel differences:

$$
\begin{gathered}
m(x, y)=\sqrt{[L(x+1, y)-L(x-1, y)]^{2}+[L(x, y+1)-L(x, y-1)]^{2}} \\
\theta(x, y)=\tan ^{-1}\left[\frac{L(x, y+1)-L(x, y-1)}{L(x+1, y)-L(x-1, y)}\right]
\end{gathered}
$$

After having assigned an image location, scale, and orientation to each keypoint, the gradient magnitude and the orientation are supplemented to a local $2 \mathrm{D}$ coordinate system, which can describe the local image region and provide these parameters with invariance [8]. The image gradient magnitude and orientation are sampled around the keypoint location. An orientation histogram is formed from the gradient orientations with sampled points within a region around the keypoint. The orientation histogram 
covers a range of orientations of $360^{\circ}$. Each sample added to the histogram is weighted by its gradient magnitude and a Gaussian-weighted circular window, with a $\sigma$ being 1.5 times of the scale of the keypoint [9]. The descriptor is formed from the vector containing the values of all the orientation histogram entries. In order to achieve orientation invariance, the coordinates of the descriptor and the gradients are rotated relative to the keypoint orientation. For each keypoint, there are 16 array of histograms with 8 orientation bins in each and 128 element feature vectors, as shown in Fig. 1.

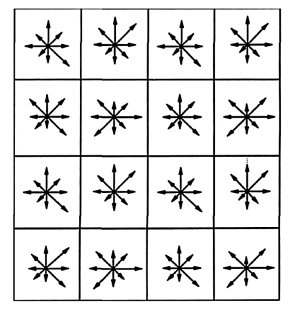

Fig. 1. SIFT keypoint descriptor

The best candidate for each keypoint is found by identifying its nearest neighbor in the database of keypoints from 2 images. The nearest neighbor is defined as the keypoint with minimum Euclidean distance for the invariant keypoint descriptor. Comparing the distance of the closest neighbor to that of the second-closest neighbor and supposing that the division of the closest neighbor to the second-closest neighbor is smaller than the global threshold, these 2 keypoints can be taken as the matched points. Otherwise, these 2 points are not matched [9].

\subsection{Epipolar Line Constraint}

For Binocular stereo vision, the two feature-matched points should locate in the same epipolar line. Using this idea, mismatched points can be found with the imaging formula [10]:

$I_{i}=\left[\begin{array}{c}u_{i} \\ v_{i} \\ 1\end{array}\right]=\left[\begin{array}{llll}k_{x i} & 0 & u_{0 i} & 0 \\ 0 & k_{y i} & v_{0 i} & 0 \\ 0 & 0 & 0 & 1\end{array}\right]\left[\begin{array}{cc}R_{i} & \mathrm{p}_{i} \\ 0 & 1\end{array}\right]\left[\begin{array}{c}x_{w} \\ y_{w} \\ z_{w} \\ 1\end{array}\right]=\left[\begin{array}{ll}M_{3 i} & \mathrm{~m}_{3 i}\end{array}\right]\left[\begin{array}{c}x_{w} \\ y_{w} \\ z_{w} \\ 1\end{array}\right]$

where, $\left(u_{i}, v_{i}\right)$ represents the coordinate on the image plan $i .\left(u_{0 i}, v_{0 i}\right)$ is the coordinate of the camera in the image plan $i .\left(k_{x i}, k_{y i}\right)$ represents the magnification in an image plan. $R_{i}$ is the $3 \times 3$ rotation matrix of camera $i$. Vector $p_{i}$ is the position vector of camera $i .\left(x_{w}, y_{w}, z_{w}\right)$ is the coordinate of the target in the world coordinate system. $M_{3 i}$ is a $3 \times 3$ matrix and $m_{3 i}$ is a $3 \times 1$ vector. For surveillance using binocular stereo vision, $i$ equals to 1 or 2 . Simplifying the target position by using the formula above for camera 1 and 2, an equation could be obtained: 


$$
I_{1}-M_{31} M_{32}^{-1} I_{2}=-M_{31} M_{32}^{-1} m_{32}+m_{31}
$$

Suppose the vector $\left[\begin{array}{lll}m_{x} & m_{y} & m_{z}\end{array}\right]^{T}=m_{31}-M_{31} M_{32}^{-1} m_{32}$, with the basic propriety of the matrix, there exists $m^{+}=\left[\begin{array}{ccc}0 & -m_{z} & m_{y} \\ m_{z} & 0 & -m_{x} \\ -m_{y} & m_{x} & 0\end{array}\right]$, so that $m^{+}\left[\begin{array}{c}m_{x} \\ m_{y} \\ m_{z}\end{array}\right]=0$.

Equation 5 can be multiplied on left by $\mathrm{m}^{+}$and simplified as:

$$
m^{+} I_{1}-m^{+} M_{31} M_{32}^{-1} I_{2}=0
$$

Equation 6 can be multiplied on left by $I_{1}^{T}$ and simplified as:

$$
I_{1}^{T} m^{+} M_{31} M_{32}^{-1} I_{2}=0
$$

Equation 6 is the epipolar line constraint equation. Denoting $F=m^{+} M_{31} M_{32}^{-1}$, which is usually called fundamental matrix, Eq. 6 will be simplified as:

$$
I_{1}^{T} F I_{2}=0
$$

\section{Aircraft Matching on an Aerodrome}

Using the algorithm of SIFT to process the images from the airport surveillance, the simulation shows that most of the keypoints locate in such areas of aircraft as: head, nose landing gear (NLG), main landing gear (MLG), inlet of the engine, vertical tail, and wingtip.

For example, using an image size of $486 \times 729$, an 8-bit grayscale, and a global threshold set 0.4 or 0.9 , results are shown in Fig. 2.

When the global threshold is set to 0.4 , there are 44 matched points, with only 1 mismatched point, and about $93 \%$ of the matched points locate on the aircraft. When the global threshold is set to 0.9 , there 93 matched points, with 22 mismatched points, and about $52 \%$ of matched points locate on the aircraft.

The global threshold is crucial, because the amount of matched points are not enough to describe the surveillance situation when the global threshold is a small value. When the global threshold is great, matched points will locate on the lamp of the apron, or vehicles, etc. These matched points are not located on the aircraft, and they are a kind of 'noise', which should be filtered out.

For civil aviation surveillance, especially for A-SMGCS policy, the method combines the algorithm of SIFT and the epipoplar line constraint for finding the mismatched points and remove them. As the error of the calibration, the matched points cannot always satisfy the epipoplar line constraint Eq. 7. To solve this problem, we define an operator $I_{1}^{T} F I_{2}$, and use this operator to calculate all matched points. The fundamental matrix is calculated during the calibration when the 2 camera are fixed. There will exist a threshold called critical threshold and all mismatched points will not 

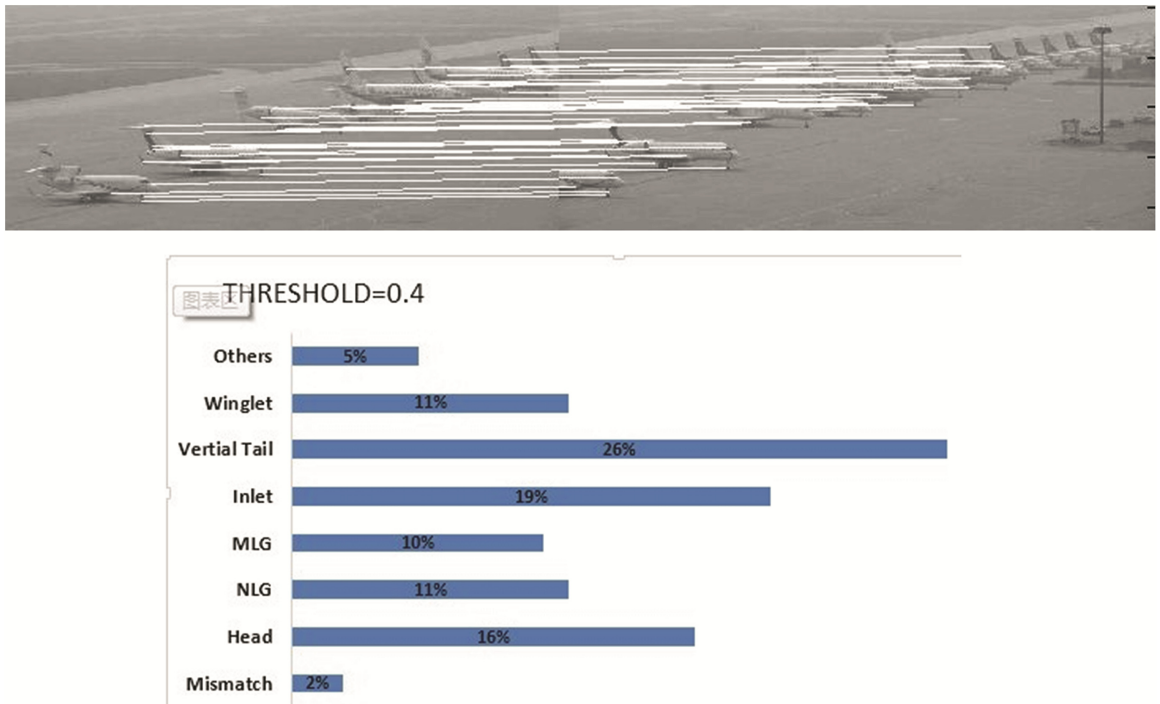

(a)

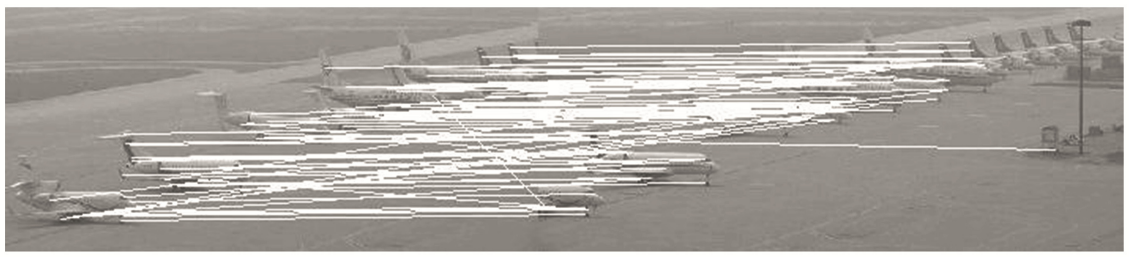

THRESHOLD $=0.9$

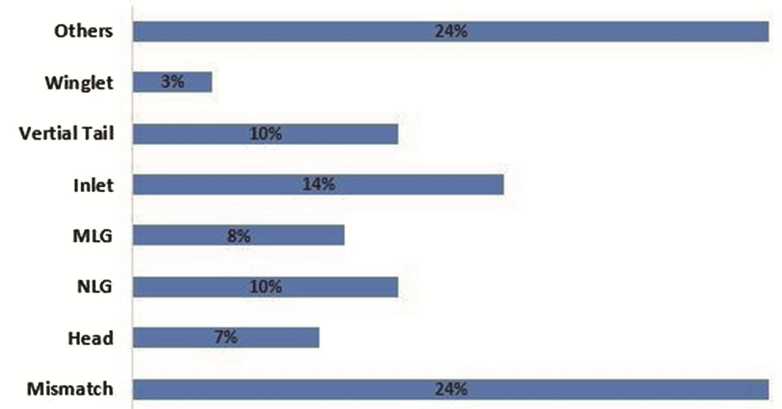

(b)

Fig. 2. (a). The statistics and the results when threshold is 0.4 (b). The statistics and the results when threshold is 0.9

satisfy the constrain $I_{1}^{T} F I_{2} \leq \varepsilon$. By this judgment, we can easily find the mismatched points and remove them.

For the same surveillance case as the previous example, 8 matched points are used to calibrate the fundamental matrix (Table 1). 
Table 1. The coordinate of 8 match points

\begin{tabular}{l|l|l}
\hline Matched point & Camera 1 coordinate & Camera 2 coordinate \\
\hline 1 & $(289.76,398.94)$ & $(295.64,565.95)$ \\
\hline 2 & $(271.71,500.62)$ & $(277.17,671.55)$ \\
\hline 3 & $(273.01,408.78)$ & $(278.44,576.18)$ \\
\hline 4 & $(245.68,529.27)$ & $(250.91,663.35)$ \\
\hline 5 & $(247.36,493.09)$ & $(277.89,498.46)$ \\
\hline 6 & $(272.47,333,73)$ & $(263.64,699.68)$ \\
\hline 8 & $(258.48,527.46)$ & $(348.44,271.92)$ \\
\hline
\end{tabular}

Putting these 8 matched points into Eq. 7, the fundamental matrix $F$ can be found as:

$$
F=\left(\begin{array}{ccc}
-7.845 \times 10^{-6} & -6.356 \times 10^{-6} & 0.011738036 \\
3.973 \times 10^{-6} & 1 \times 10^{-7} & 0.003476968 \\
-0.005120125 & -0.002781841 & 0.0476961799
\end{array}\right)
$$

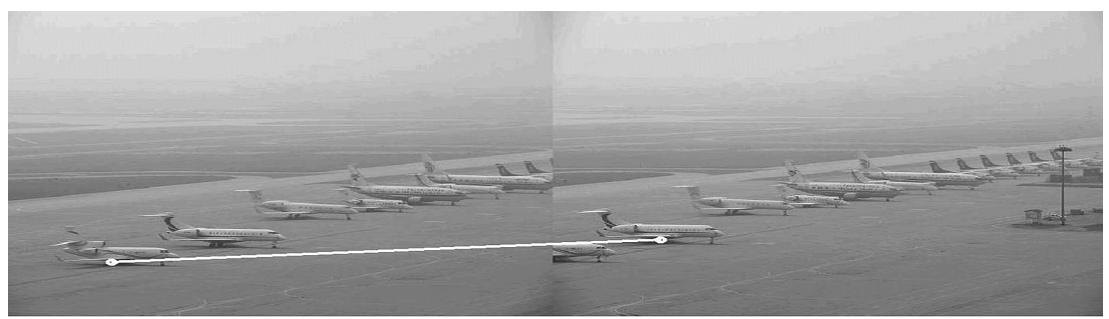

(a)

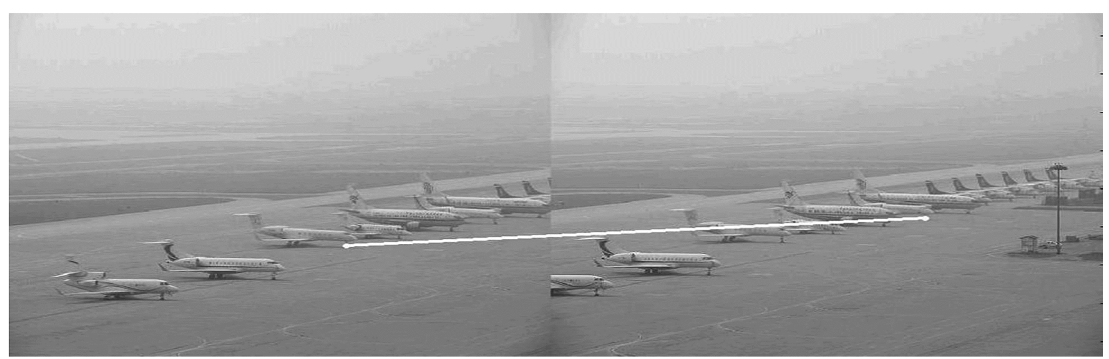

(b)

Fig. 3. (a). Mismatched point 1 (b). Mismatched point 2 
When the global threshold is set to $0.7,73$ matched points are obtained by calculating the operator $I_{1}^{T} F I_{2}$, and two mismatched points are also obtained, shown in Fig. 3:

$$
\begin{aligned}
& I_{21}=(398.97,160.65), I_{11}=(356.10,139.28), \text { with } I_{11}^{T} F I_{21}=1.046 \\
& I_{22}=(324.48,495.09), I_{12}=(288.34,486.61), \text { with } I_{12}^{T} F I_{22}=1.233
\end{aligned}
$$

The matched point calculated by the operator $I_{1}^{T} F I_{2} \in[0.39,0.53]$. In this case, the critical threshold is defined as $\varepsilon=0.8$, since the result calculated by the operator of the mismatched point is much bigger than the matched point. Checking by visual inspection, the result is correct.

\section{Conclusion}

Combining the SIFT and the epipolar line constrain, the method is for target matching of airport surveillance. The global threshold should not be great when targets to be positioned are the aircraft. Because of the error of calibration, the epipolar line constrain equation can't be used directly. Therefore an operator $I_{1}^{T} F I_{2}$ and the critical threshold should be defined and all matched points will satisfy the condition that the absolute value of $I_{1}^{T} F I_{2}$ is no large than $\varepsilon$. The simulation shows that this algorithm works well for the aircraft matching for airport surveillance.

\section{References}

1. Campbell, S.D., Londner, E., Keefe, J., Edwards, C.: Airport surveillance requirements validation using Monte Carlo simulation. In: Digital communications - Enhanced Surveillance of Aircraft and Vehicles (TIWDC/ESAV), Tyrrhenian International Workshop on, Roma, Italy, IEEE (2014)

2. Luo, X., Lu, Y., Wu, H.G.: A novel airport surface surveillance method using multi-video fusion. Telecommun. Eng. 7, 128-132 (2011)

3. Zeng, L., Wang, Y., Tan, J.: Improved algorithm for SIFT feature extraction and matching. Opt. Precis. Eng. 5, 1391-1397 (2011)

4. Cheng, D.Z., Li, Y.J., Yu, R.X.: Image matching method based on improved SIFT algorithm. 17, 285-287 (2011)

5. Lindeberg, T.: Scale-space theory: a basic tool for analyzing structures at different scales. J. Appl. Stat. 21(2), 225-270 (1994)

6. Hsu, C.-Y., Lu, C.-S., Pei, S.-C.: Image feature extraction in encrypted domain with privacypreserving SIFT. IEEE Trans. Image Process. 21, 4593-4607 (2012)

7. Wang, H., Yang, K., Gao, F., Li, J.: Normalization methods of SIFT vector for object recognition. In: 2011 Tenth International Symposium on Distributed Computing and Applications to Business, Engineering and Science (DCABES), Wuxi, China. IEEE (2011)

8. Lowe, D.G.: Object recognition from local scale-invariant features. In: International Conference of Computer Vision, pp. 1150-1157 (1999) 
9. Lowe, D.G.: Distinctive image features from scale-invariant keypoints. Int. J. Comput. Vision 60(2), 91-110 (2004)

10. Sonka, M., Hlavac, V., Boyle. R.: Image Processing, Analysis, and Machine Vision, 3rd edn. 